parallel to the principal axis are very different, in magnesium they are almost the same ${ }^{1}$.

With regard to Prof. Herrmann's second point ${ }^{2}$, that the eccentricity of the ellipsoidal space occupied by an atom in zinc or cadmium is almost exactly $1 / 2$, and that into this space one can place two spheres in contact with their centres at the foci, it is difficult to see what physical significance this geometrical result can have ; if one regards the atom as occupying alternately the two foci, the situation is equivalent to a larger vibration along the $c$-axis than normal to it.

Finally, it may be mentioned that since my letter appeared in NATURE, the results for zine have been discussed in the Philosophical Magazine ${ }^{2}$, and a preliminary account of the cadmium results has appeared in the Proceedings of the Leeds Philosophical Society ${ }^{3}$.

Physics Laboratories, G. W. BRINDLEY.

University, Leeds. July 13.

${ }^{1}$ See, for example, a recent paper by E. Goens and E. Schmid, Phys. Z., 37, 385 (1936)

${ }^{2}$ Phil. Mag., 21, 790 (April 1936)

3 Proc. Leeds Phil. Soc., 3, 200 (April 1936).

\section{Oxide Layer on a Polished Surface}

I CAN confirm S. Dobinski's results ${ }^{1}$ for copper by my own experiments on polished aluminium ${ }^{2}$. I used a different method, namely, observations of changes in the initial solution potential when polished under paraffin, benzene, water. The conclusion I came to in my paper was that wet "polishing resulted in the formation of an oxide film in close contact with the metal and similar to that formed by heating in air". This seems further evidence that many observations on the so-called 'amorphous' layer may really have been observations on the properties of the oxide.

16 Sandileigh Avenue, L. H. Callendar.

Withington,

Manchester.

1 NATURE, 138, 31, July 4, 1936.

" "The Influence of Boundary Films on Corrosive Action", Proc. Roy. Soc., A, 115, 368 (1927).

Inhibitive Effect of Vitamin C on Toxin Production by $C$. diphtheria

KING and Menten (1935) have reported that guinea-pigs having a high vitamin $\mathrm{C}$ storage are less sensitive to diphtheria toxin than are those having a low vitamin $\mathrm{C}$ storage. This observation may be important in relation to the pathogenesis of diphtheria infection. We, therefore, tried to ascertain the effect of vitamin $\mathrm{C}$ on toxin production by $C$. diphtheria in vitro.

Experiments have been conducted on the influence of vitamin $\mathrm{C}$ in the culture media on the toxicity of culture filtrates of a toxin-producing strain of C. diphtheria. A sterile solution of vitamin $\mathrm{C}$ was added to the culture medium in concentrations ranging from 0.05 to 0.125 per cent. The flasks containing vitamin as well as corresponding controls were inoculated with $C$. diphtheria, incubated 48 hours at $30^{\circ} \mathrm{C}$., and the toxicity of the filtrates tested by intracutaneous injection in guinea pigs.

Vitamin $\mathrm{C}$ added to culture media rapidly dissppears on incubation at $30^{\circ}$ or $37^{\circ} \mathrm{C}$. However, if immediately after the vitamin is added the medium is inoculated with $C$. diphtheria, the vitamin dissppears more slowly. But most significant is the fact that even small residual amounts of the vitamin (20-40 mgm. per cent) are sufficient to inhibit toxin production.

A series of experiments under a variety of conditions has clearly established the fact that vitamin $\mathrm{C}$ added in small amounts to a suitable medium inhibits toxin production. The same strain of $C$. diphtheria produces a potent toxin in the flask without vitamin $\mathrm{C}$, and little or no toxin in the one containing the same medium plus the addition of 0.05 per cent ascorbic acid.

It is suggested that the varying toxic character of a diphtheritic injection may depend as much on the tissue saturation with vitamin $\mathrm{C}$ as on the toxigenic properties of the infecting strain. It is also possible that the mildness of diphtheritic infection in subtropical and tropical countries is in some way related to the quantitative differences in the concentration of this vitamin. These questions are being investigated.

Department of Hygiene and Bacteriology,

I. J. KLIGLer. Hebrew University, Jerusalem.

\section{Induced Chiasma Formation in Somatic Cells by a Carcinogenic Hydrocarbon}

Experiments are being done on the effects of methylcholanthrene on mouse fibroblasts cultivated in vitro. The tissue is grown by the hanging drop method in a medium containing approximately $0.005 \mathrm{mgm}$. of the hydrocarbon per c.c. and is subcultivated every 48 hours. After three days' growth, the cultures when fixed in Navashin's fluid and stained with iodine-gentian-violet are found to contain a high percentage of abnormal mitotic figures of various types.

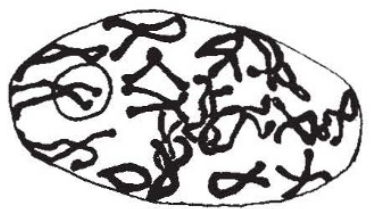

FIG. 1.

Of particular cytological interest is the induction of chromosome pairing and chiasma formation in somatic cells. This condition was observed in several tetraploid cells at various stages from early prophase until anaphase. This disturbance may take the form of a precocity of the prophase in relation to the chromosome splitting, which may also be the underlying mechanism of the induction of chromosome pairing. This suggestion is supported by the abnormal degree of contraction which is observed in the chromosomes both in the cells which show chiasma formation and in the other abnormal cells. The chiasma frequency observed is 1-3 per chromosome as in normal meiosis. A typical prophase cell of this type is shown in the accompanying drawing (Fig. 1) in which some chromosomes have been omitted for clarity.

E. Marie Hearne.

(Canadian Federation of University Women's Scholar.)

Strangeways Research Laboratory, Cambridge. July 16. 